\title{
Optokinetic nystagmus in patients with central scotomas in age related macular degeneration
}

\author{
C Valmaggia, J Charlier, I Gottlob
}

\begin{abstract}
Background-Reports on the impact of a loss in the central field of vision on optokinetic nystagmus (OKN) are varied. A study was therefore undertaken to reassess the role of the central retina in the generation of $\mathrm{OKN}$ in a large group of patients with age related macular degeneration.

Methods-Four groups of 20 patients were examined: a control group without scotoma and three groups with absolute central scotomas measuring $1^{\circ}-10^{\circ}, 11^{\circ}-$ $20^{\circ}$, and $21^{\circ}-30^{\circ}$. OKN was elicited with black and white stripes moving nasally to temporally or temporally to nasally on a screen subtending $54^{\circ} \times 41^{\circ}$ at four velocities $\left(15,30,45\right.$, and $\left.60^{\circ} / \mathrm{s}\right)$. OKN gain was measured using infrared oculography.

Results-There was no significant difference in OKN gain between the control group and those with scotomas of $1^{\circ}-10^{\circ}$ and $11^{\circ}-20^{\circ}$. A significant difference in OKN gain was found between the group with scotomas of $21^{\circ}-30^{\circ}$ and all other groups at stimulus velocities of 30,45 , and $60 \%(\mathrm{p}<0.05)$. OKN gain significantly diminished with increasing stimulus velocity $(p<0.05)$. No statistically significant difference was found in OKN gain between stimuli moving temporally to nasally and nasally to temporally.

Conclusion-Abnormalities of $\mathrm{OKN}$ gain were noted only in patients with large scotomas. An intact macula is therefore not necessary for the generation of $O K N$. (Br f Ophthalmol 2001;85:169-172)
\end{abstract}

The optokinetic nystagmus (OKN) combines with the vestibulo-ocular reflex and the smooth pursuit system in the task of retinal image stabilisation. ${ }^{1}$ The pattern of OKN consists of a slow component in the direction of the target movement and a fast component in the opposite direction. ${ }^{2}$ The effectiveness of $\mathrm{OKN}$ in reducing retinal image slip may be indicated by the ratio of slow phase eye velocity to stimulus velocity which is termed the gain. The impact of a loss of the central visual field on $\mathrm{OKN}$ is controversially discussed in the literature. In subjects with artificially produced central scotomas both a decrease $\mathrm{e}^{3-5}$ and an increase $^{6}$ in OKN gain have been described. In patients with central scotomas in age related macular degeneration a slight insignificant diminution of $\mathrm{OKN}$ gain $^{2}{ }^{7}$ and an increase in OKN gain $^{89}$ have been reported. Because of these discrepancies, this study was designed to reassess the role of the central retina in the generation of $\mathrm{OKN}$ by investigating a large group of patients classified according to the size of their central scotoma in age related macular degeneration.

\section{Patients and methods}

PATIENTS

Each patient had a complete ophthalmological examination including simultaneous fluorescein and indocyanin green angiography. The size of the absolute scotoma was measured with the mark Ie $4\left(1 / 4 \mathrm{~mm}^{2}\right.$ and $\left.318 \mathrm{~cd} / \mathrm{m}^{2}\right)$ of the Goldmann perimetry using a standard background luminance $\left(10 \mathrm{~cd} / \mathrm{m}^{2}\right)$. Only one eye per patient was tested. Four different groups of 20 patients were investigated: a control group with macular degeneration but without scotoma (age range $57-86$ years; mean 71.4 years; mean distance visual acuity $20 / 30$ ), a group with central scotomas of $1^{\circ}-10^{\circ}$ (age range $57-87$ years; mean 74.9 years; mean distance visual acuity 20/60; duration of visual symptoms: range 1-18 months; mean 5.5 months), a group with central scotomas of $11^{\circ}-20^{\circ}$ (age range 65-94 years; mean 77.4 years; mean distance visual acuity 20/300; duration of visual symptoms: range 5-24 months; mean 12.2 months), and a group with central scotomas of $21^{\circ}-30^{\circ}$ (age range 62-91 years; mean 73.8 years; mean distance visual acuity 20/600; duration of visual symptoms: 12-34 months; mean 22.8 months). There was a highly significant correlation between the duration of visual symptoms and the size of scotoma in the whole group of patients $(p<4 \times$ $\left.10^{-14}\right)$. The patients in the control group had angiographically early age related macular degeneration with either drusen or pigment epitheliopathy, and those in the three groups with scotomas showed advanced age related macular degeneration with subfoveolar choroidal neovascularisation. They had no history of strabismus or amblyopia. Patients with glaucoma or diabetic retinopathy were excluded from the study.

The study was approved by the ethics commission of the Kantonsspital St Gallen. Informed consent was obtained from all patients after explanation of the nature of the investigation.

\section{METHODS}

OKN stimulation and eye movement recordings and analysis were performed with vision monitor equipment (Metrovision, Perenchies, France). Visual stimuli were generated on a colour monitor measuring $51 \mathrm{~cm}$ diagonally placed $40 \mathrm{~cm}$ away from the eyes with a frame rate of $120 \mathrm{~Hz}$. The screen covered a visual 


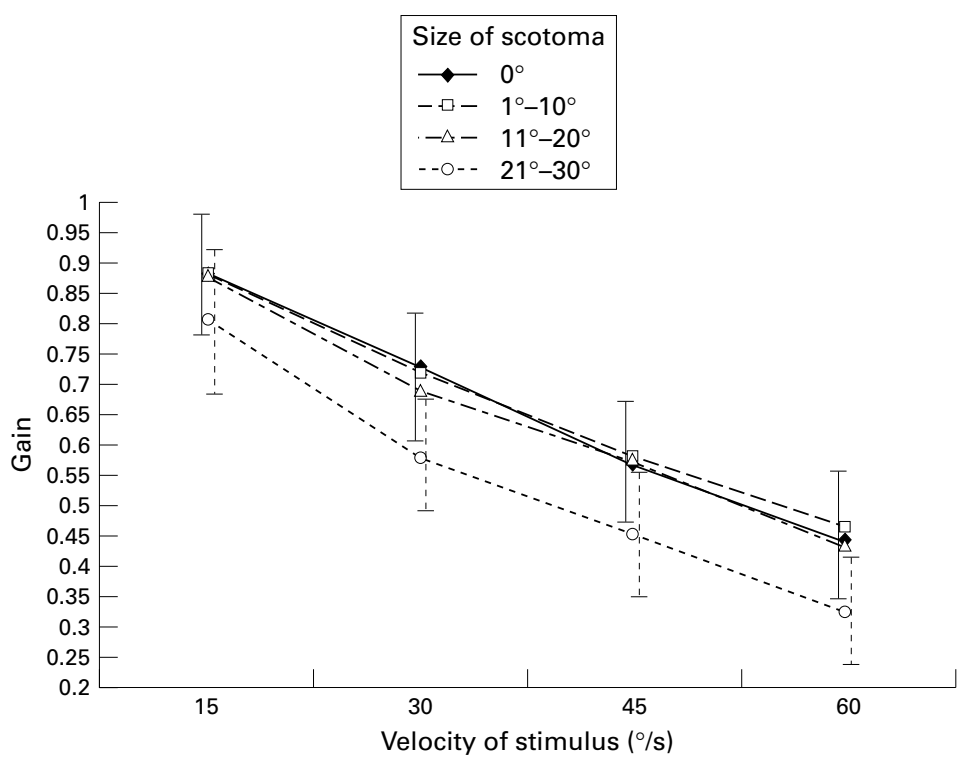

Figure 1 Mean (SD) OKN gain during stimulation from temporal to nasal with stripes moving at $15,30,45$, and $60^{\circ} /$ measured from eyes without scotomas or with scotomas of $1^{\circ}-10^{\circ}, 11^{\circ}-20^{\circ}$, and $21^{\circ}-30^{\circ}$.

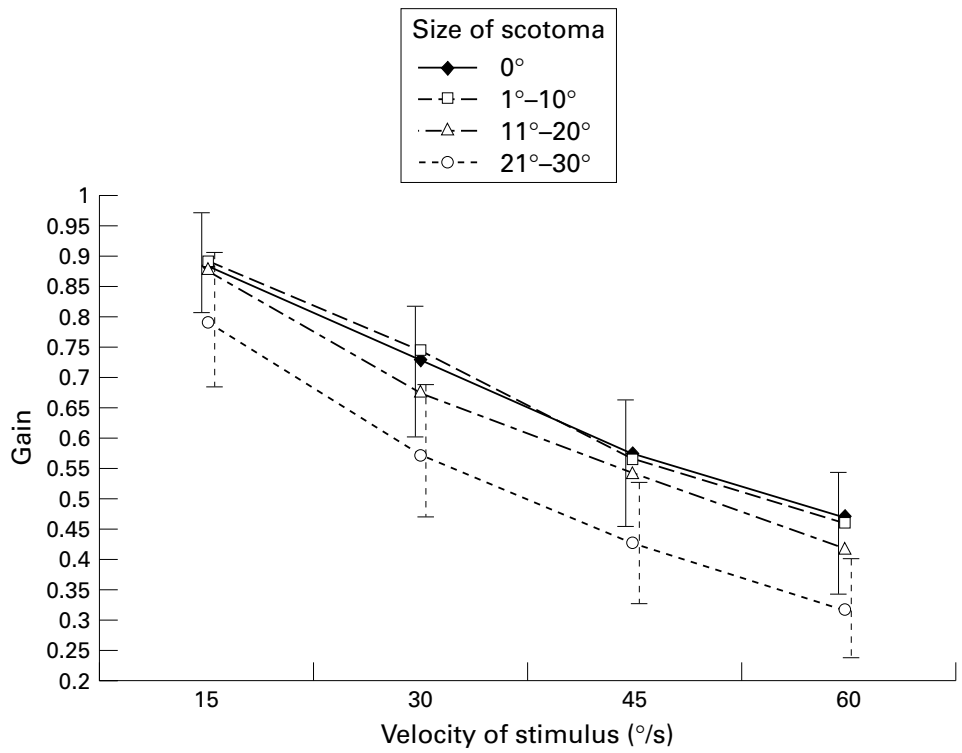

Figure 2 Mean (SD) OKN gain during stimulation from nasal to temporal with stripes moving at 15,30,45, and $60^{\circ} /$ measured from eyes without scotomas or with scotomas of $1^{\circ}-10^{\circ}, 11^{\circ}-20^{\circ}$, and $21^{\circ}-30^{\circ}$.

field of $54^{\circ}$ horizontally and $41^{\circ}$ vertically. OKN was elicited with alternating white vertical stripes of luminance $70 \mathrm{~cd} / \mathrm{m}^{2}$ and black vertical stripes of luminance $<1 \mathrm{~cd} / \mathrm{m}^{2}$. Each stripe covered $2^{\circ}$ of visual angle (equivalent to a visual acuity of 20/2400). The stripes moved at a constant velocity of $15,30,45$, or $60 \%$, either nasally to temporally or temporally to nasally, for 40 seconds in random order. One eye of each patient was investigated monocularly at all four stimulus velocities and both directions with a time interval of 2 minutes between the trials. Patients were instructed not to follow individual stripes across the visual field but to attempt to fixate stripes as they passed in front of them.

Eye movements were recorded by measuring the position of the corneal reflex with respect to the centre of the pupil. The investigation was independent of head movements. A near infrared illumination of the eye $(880 \mathrm{~nm})$ was used to produce the corneal reflex and the pupil image. The system operated with a sampling rate of $60 \mathrm{~Hz}$ and achieved a resolution of 10 arc minutes. Eye movement analysis included the detection of OKN slow and fast phases such as the determination of the average velocity for the slow phases. Five seconds after stimulus onset the mean velocity of consecutive slow phases was measured for each stimulus velocity and stimulus direction during a period of 10 seconds. This analysis was carried out without knowledge of the clinical data of the patients. The OKN gain, defined as the ratio of slow phase velocity to stimulus velocity, was measured and the results were analysed by analysis of variance using the StudentNewman-Keuls test. A difference in OKN gain was considered significant at a $p$ value of $<0.05$.

\section{Results}

Mean OKN gains for stimulation from temporal to nasal are shown in Figure 1 and from nasal to temporal in Figure 2. The difference in $\mathrm{OKN}$ gain between the control group, the group with central scotomas of $1^{\circ}-10^{\circ}$, and the group with central scotomas of $11^{\circ}-20^{\circ}$ was not significant. The difference in $\mathrm{OKN}$ gain was significantly lower in the group with central scotomas of $21^{\circ}-30^{\circ}$ compared with all other groups at stimulus velocities of 30,45 , and $60 \%(\mathrm{~s}<0.05)$; there was no significant difference between the groups at a stimulus velocity of $15 \%$ s. In each group the $\mathrm{OKN}$ gain significantly diminished for each increase in stimulus velocity $(p<0.05)$. No difference was found in OKN gain between movement of the stimulus temporally to nasally or nasally to temporally in any group.

\section{Discussion}

Our study has shown, in a large group of 60 patients with central scotomas caused by age related macular degeneration of $1^{\circ}-30^{\circ}$ compared with a control group of 20 patients without scotomas, that an intact central retina is not essential to generate $\mathrm{OKN}$.

A statistically significant reduction in $\mathrm{OKN}$ gain was found only for central scotomas of more than $20^{\circ}$ at stimulus velocities of 30,45 , and $60 \%$. These results must be interpreted by considering the role played by the area of the visual field used to generate OKN. Dichgans et $a l^{10}$ found that OKN gain did not decrease significantly if the vertical angular size of the visual field used for stimulation was reduced to $2^{\circ}$, but that $\mathrm{OKN}$ gain was significantly reduced when the horizontal angular size, as in the stimulation field used in our investigations, was below $60^{\circ}$. Furthermore, they showed that the correlation between OKN gain and angular size was most marked at increasing velocity. It is therefore possible that, if we had used a larger stimulation field, no reductions in $\mathrm{OKN}$ gain would have been observed, even for scotomas of more than $20^{\circ}$.

Our findings confirm the non-significant lowering of $\mathrm{OKN}$ gain for central scotomas 
reported by most authors. A slightly reduced OKN gain was reported by Yee et al in six patients with central scotomas of $5^{\circ}-15^{\circ}$, by Abadi and Pantazidou ${ }^{7}$ in six patients with central scotomas of $1^{\circ}-12^{\circ}$, and by van Die and Collewijn ${ }^{13}$ in three patients with central scotomas of $5^{\circ}-10^{\circ}$. These results differ from studies using artificial central scotomas which reported a large reduction in OKN gain even for small scotomas. ${ }^{3-5} 11-13$ However, in experiments with retinal stabilised scotomas the OKN can introduce a source of error as the scotoma itself is seen to move and can become a stimulus for further movement or can be used to stare at while the moving stripes are neglected. ${ }^{11}$ In studies with artificial scotomas the edge effect of the mask used to create the central field loss is also likely to have contributed to the suppression of the OKN. When stationary edges were eliminated $\mathrm{Mu}-$ rasugi et $a l^{14}$ found no significant reduction in OKN gain during occlusion at low stimulus velocities. Similarly, the OKN gain for scotomas of more than $20^{\circ}$ was not significantly reduced at a slow stimulus velocity of $15 \%$ in our study. This is also in agreement with Howard and $\mathrm{Ohmi}^{15}$ using occlusion of the central retina.

Another difference between central scotomas in age related macular degeneration and artificial central scotomas may be caused by the difference in duration of the scotoma in the two groups. It is possible that subjects with a longstanding scotoma have established compensatory mechanisms such as different fixation patterns or filling in and therefore have better OKN responses than subjects with an acute artificial scotoma. The duration of the visual symptoms correlates strongly with the size of the scotomas in our study. As these two parameters are interdependent and OKN gain is related to the size of the scotoma, it is therefore not possible to conclude from our data whether the duration of visual symptoms influences the OKN gain independently of the size of the scotoma.

In normal subjects $\mathrm{OKN}$ gain decreases with increasing stimulus velocity ${ }^{10} 16$ and with ageing. ${ }^{18-20}$ We found a statistically significant diminution in OKN gain at each increase in velocity in our control group and in the three groups with scotomas. Our findings in the control group related to the age of the patients were also consistent with the results reported in the literature.

In the first few weeks after birth infants show a strong monocular OKN asymmetry. ${ }^{21}{ }^{22}$ The response to temporal to nasal stimulation is present while the response to nasal to temporal stimulation is weak or absent at birth. Simultaneously with the development of binocular vision, this $\mathrm{OKN}$ asymmetry disappears. In patients with abnormal development of binocularity such as strabismus or amblyopia, OKN asymmetries persist later in life. ${ }^{23}$ This supports the view that an intact and functionally normal fovea during early infancy is necessary to develop a symmetrical monocular OKN. ${ }^{24-27}$ Our results confirm that scotomas acquired later in life do not modify the developed symmetry.

Interestingly, OKN stimulation covering $54^{\circ}$ horizontally and $41^{\circ}$ vertically of the visual field was sufficient to obtain results similar to those obtained by Yee et $a l^{2}$ and Abadi and Pantazidou ${ }^{7}$ who used full field OKN stimulation. This confirms the findings of Howard and $\mathrm{Ohmi}^{15}$ that high gain OKN may be elicited from narrow moving displays and that the traditional type of large optokinetic drum which rotates around a subject is not the only method of investigating OKN accurately.

In conclusion, we have shown that scotomas smaller than $20^{\circ}$ do not significantly alter $\mathrm{OKN}$ gain and have confirmed in a larger group of patients the results reported by Yee et $a l^{2}$ and Abadi and Pantazidou. ${ }^{7}$ We have also confirmed previous reports of Howard and $\mathrm{Ohmi}^{15}$ that higher stimulus velocities of OKN are more susceptible to being altered with central scotoma.

1 Westheimer G, McKee S. Visual acuity in the presence of retinal-image motion. F Opt Soc Am 1979;65:847-50.

2 Yee R, Baloh R, Honrubia V, et al. Pathophysiology of optokinetic nystagmus. In: Honrubia V, Brazier M, eds. Nystagmus and vertigo. London: Academic Press, 1982: $251-75$.

3 Cheng $\dot{M}$, Outerbridge J. Optokinetic nystagmus during selective retinal stimulation. Exp Brain Res 1975;23:129-39.

4 Dubois M, Collewijn H. Optokinetic reations in man elicited by localised retinal motion stimuli. Vision Res 1979; 19:1105-15.

5 Van Die G, Collewijn H. Optokinetic nystagmus in man. Role of central and peripheral retina and occurence of symmetries. Human Neurobiol 1982;1:111-9.

6 Gresty M, Halmagyi M. Following eye movements in the absence of central vision. Acta Otolaryngol 1979;87:47783.

7 Abadi R, Pantazidou M. Monocular optokinetic nystagmus in humans with age-related maculopathy. $\mathrm{Br} \mathcal{F}$ Ophthalmol 1997;81:123-9.

8 Hood J. Observations upon the neurological mechanism of optokinetic nystagmus with especial reference to the contribution of peripheral vision. Acta Otolaryngol 1967;63: 208-15.

9 Hood J. Observation upon the role of the peripheral retina in the execution of eye movements. F Otolaryngol 1975;37:6573 .

10 Dichgans J, Nauck B, Wolpert E. The influence of attention, vigilance and stimulus area on optokinetic and vestibular nystagmus and voluntary saccades. In: Zikmund V, ed. The oculomotor system and brain functions. London: Butteroculomotor system and
worths, 1973:279-94.

11 Gresty M, Halmagyi M. Following eye movements in the absence of central vision. Acta Otolaryngol 1979;87:47783.

12 Miyoshi T. Role of the central and peripheral retina upon optokinetic nystagmus. Acta Otolaryngol 1985;419:53-61.

13 Van Die G, Collewijn H. Control of optokinetic nystagmus by the central and peripheral retina: effects of partial visual field masking, scotopic vision and central retinal scotomata. Brain Res 1986;383:185-94.

14 Murasugi C, Howard I, Ohmi M. Optokinetic nystagmus: the effect of stationary edges, alone and in combination with central occlusion. Vision Res 1986;26:1155-62.

15 Howard I, Ohmi M. The efficiency of the central and peripheral retina in driving human optokinetic nystagmus. Vision Res 1984;9:969-76.

16 Holm-Jensen S, Peitersen E. The significance of the target frequency and the target speed in optokinetic nystagmus. frequency and the target speed in

17 Sills A, Honrubia V, Konrad H, et al. A rapid optokinetic nystagmus test: comparison with standard testing. Trans Am Acad Ophthalmol Otolaryngol 1976;82:223-31.

18 Simons B, Buttner U. The influence of age on optokinetic nystagmus. Eur Arch Psychiatr Neurol Sci 1985;234:369-73.

19 Ura M, Pfaltz C, Allum J. The effect of age on the visuo- and vestibulo-ocular reflexes of elderly patients with vertigo. Acta Otolaryngol Suppl 1991;481:399-402.

20 Baloh R, Jacobson K, Socotch T. The effect of aging on visual-vestibulo-ocular responses. Exp Brain Res 1993;95: 509-16.

21 Naegele J, Held R. The post-natal development of monocular optokinetic nystagmus in infants. Vision Res 1982;22: 341-6.

22 Lewis T, Maurer D, Smith R, et al. The development of symmetrical optokinetic nystagmus during infancy. Clin Vis Sci 1992;7:211-8.

23 Schor C, Levi D. Disturbance of small-field horizontal and vertical optokinetic nystagmus in amblyopia. Invest $O p h$ thalmol Vis Sci 1980;19:668-83. 
24 Atkinson J, Braddick O. Development of optokinetic nystagmus in infants: an indicator of cortical binocularity? In Fisher D, Monty R, Senders J, eds. Eye movements: cognition and visual perception. Hillsdale, NJ: Erlbaum, 1981:53-64. 25 Naegele J, Held R. The postnatal development of monocular optokinetic nystagmus in infants. Vision Res 1982;22:341-6.
26 Mohn G, Sireteanu R, van Hof-van Duin J. The relation of monocular optokinetic nystagmus to peripheral binocular interactions. Invest Ophthalmol Vis Sci 1986;27:565-73.

27 Weinacht S, Kind C, Schulte J, et al. Visual development in preterm and full-term infants: a prospective masked study. Invest Ophthalmol Vis Sci 1999;40:346-53.

\section{Cover illustration: Double vision in an aerial dynamo}

The vigilant white fronted bee eater (Merops bullockoides) shown on the cover has chosen a perch with an unobstructed view of the surroundings. From there, she is able to scan the near horizon for a meal. Here, the elegant high stakes drama between predator and prey begins with the location of an insect, erratically flying, often against a variably shaded background. While she can find insects crawling on tree branches or leaves, she seems to prefer and is exquisitely prepared for an aerial ballet. The chase begins with uniocular tracking and the bee eater uses her more nasal fovea until the range is close and thus requires stereopsis. She then turns her head slightly and the image swings via the infula to the temporal foveae in both eyes allowing for acute depth perception in the three dimensional environment. As the distance closes, the bee eater may choose her favourite strategy to avoid the bee's stinger. The bird has learned to capture, kill, and devour truly dangerous, and even venomous, insects such as wasps without getting stung, by approaching the insect on the perpendicular taking the wasp across the abdomen, often from below. She will return to a favourite perch, settle on it, and then smack the wasp, head first, against an adjacent branch, thus dispatching it. Then she scrapes the stinger from the rear of the insect and swallows the insect.

These magnificent predators are wing feeders and have one of the most difficult hunting tasks in the animal kingdom. Evolution has given her the engineering equipment necessary to manage such a task. Some bee eaters have been documented to see flying insects at 100 metres while on the wing! But this remarkable acuity is only a small part of the story. The dual foveae allow for these birds to establish uniocular fixation and, when ready, to slip the image along the infula (see BFO August 2000 cover description $(2000 ; 84: 821)$ for discussion) to the temporal fovea allowing for coordination with the contralateral eye, and creating stereopsis with that contralateral fovea. But the elegance and speed of this capture is much more thrilling to watch than this description, for much more must go into it. The retinal processing must be more rapid than our own (many such species have three times the concentration of amacrine cells in their retina to allow for such processing). The cerebellar coordination must similarly be rapid and precise, for the muscular requirements need be as precise as those of a retinal surgeon attempting subretinal membrane removal. The photograph on the left of the cover illustrates the alert bird on her perch, poised and scanning for insect flight. The right side of the cover illustrates the posterior segment of such a bifoveate bird. The pecten can be seen clearly as a dark structure in the lower half of the photograph, and each of two foveal pits can been seen on a horizontal line above the pecten. The pit on the left is the more nasal "uniocular" fovea. The foveal pit on the right is the more temporal fovea and accomplishes stereopsis with the contralateral temporal fovea. The infula is not illustrated in this photograph, but is present in the bee eaters.

The natural history of this brightly decorated bird is as sociologically interesting as is its visual mechanisms. This bird is a cooperatively breeding species and is the subject of ongoing investigation by ornithologist, Steven Emlen. During the breeding season these birds live in colonies of 200 or more and aggregate in clans of related birds. The clans will break up into monogamous pairs, with one or more male helpers. This assistant male will help construct the nest, help with nest defence, and with the provision of food for the brooding female. These males are related and usually from the previous year's brood. Despite being sexually mature these birds do not breed as helpers. Surprisingly, this is an evolutionary strategy which will assist these birds individually and cooperatively. That it helps the monogamous pair goes without saying, especially with predator protection and acquisition of food, but this strategy also helps the nonbreeding "helper". Often, nesting sites in the soil banks of local rivers are at a premium and construction of these nests is a learned behaviour. The helper will have a better chance of inheriting the territory or belonging to a clan that will together invade a new territory. Emlen has observed that these bee eaters live in a harsh environment and this cooperative breeding improves survivability, since breeding as a pair without help involves a high cost and a low probability of success. The presence of a helper ensures an average of one additional offspring for every two nesting attempts-a very high benefit ratio for having a helper.

The white fronted bee eater is a very well adapted social species that has one of the most acute visual systems on earth. She is probably not troubled by her "double vision."-Ivan R SCHWAB, MD, UC Davis Department of Ophthalmology, 4860 Y Street, Suite 2400, Sacramento, CA 95817,USA (irschwab@ucdavis.edu). 